Check for updates

Cite this: RSC Adv., 2017, 7, 32842

Received 19th March 2017

Accepted 20th June 2017

DOI: 10.1039/c7ra03221h

rsc.li/rsc-advances

\section{Preparation, characterization and in vitro cell performance of anti-washout calcium phosphate cement modified by sodium polyacrylate}

\begin{abstract}
Xingmei Li, ${ }^{\text {ab }}$ Fupo $\mathrm{He}^{\mathrm{c}}$ and Jiandong Ye (D) *abd
The anti-washout ability of calcium phosphate cement (CPC) is essential for its application in massive hemorrhage regions. However, the undesirable anti-washout property of CPC inhibits its broader application. This study reports the effectiveness of sodium polyacrylate (PAAS) in the enhancement of anti-washout ability of CPC. The washout resistance, injectability, rheological property, setting time, compressive strength, phase, microstructure, and in vitro cell behaviors of the modified CPC were evaluated. The results showed that PAAS significantly enhanced the anti-washout property and injectability of the cement paste. Meanwhile, PAAS hardly affected the precipitation of hydroxyapatite (HAp), setting time, and microstructure. A low content of PAAS slightly reduced the compressive strength of CPC, but the compressive strength of CPC gradually increased with increasing PAAS content. The in vitro cell assessments demonstrated that all the samples were biocompatible and mouse bone mesenchymal stem cells (mBMSCs) well adhered, spread, and proliferated on the samples. This antiwashout CPC modified by PAAS has potential application prospects in orthopedic surgery.
\end{abstract}

\section{Introduction}

Calcium phosphate cement (CPC) was successfully created by Brown and Chow in 1980s. ${ }^{1}$ In the last three decades, CPC had been increasingly investigated and used as a bone substitute material because of its excellent biocompatibility, bioactivity, osteoconductivity, injectability, self-setting characteristic, and easy shaping for targeting specific defects. ${ }^{2}$ Injectable CPC has the potential to repair bone defects by minimally invasive surgical techniques. However, it is widely accepted that there are some crucial issues that need to be solved to satisfy clinical requirements, such as the undesirable anti-washout ability. The CPC paste tends to disintegrate due to its weak cohesion, when it contacts with biological fluids or blood in surgical sites in clinical applications. Solid particles separated from CPC may get into the blood stream, causing a severe inflammatory response, pulmonary embolism, and cardiovascular deterioration in vertebroplasty or kyphoplasty procedures. ${ }^{3}$ Therefore, it is necessary to improve the anti-washout ability of injectable CPCs to meet the demand of clinical applications.

\footnotetext{
${ }^{a}$ School of Materials Science and Engineering, South China University of Technology, Guangzhou 510641, China. E-mail: jdye@scut.edu.cn

${ }^{b}$ National Engineering Research Center for Tissue Restoration and Reconstruction, Guangzhou 510006, China

${ }^{c}$ School of Electromechanical Engineering, Guangdong University of Technology, Guangzhou 510006, China

${ }^{d}$ Key Laboratory of Biomedical Materials of Ministry of Education, South China University of Technology, Guangzhou 510641, China
}

Accelerating the setting process and improving cohesion are both effective ways to improve the anti-washout property of injectable CPCs. Many liquid or solid substances have been used to reduce setting time of CPC. Previous studies revealed that the addition of citric acid, ${ }^{4}$ mesoporous bioactive glass, ${ }^{5}$

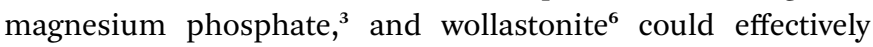
shorten the setting time and improve the anti-washout property of CPC. Moreover, increasing the cohesion of cement paste has been proved another useful strategy to improve anti-washout property. Numerous synthetic or natural polymers, such as sodium alginate, ${ }^{7}$ chitosan, ${ }^{8-10}$ modified starch, ${ }^{10}$ hydroxypropyl methylcellulose,${ }^{11-13}$ polylactide, ${ }^{14}$ and xanthan gum, ${ }^{3}$ are efficient additives for improving the cohesion of CPC paste. However, in many cases the improvement in anti-washout property of CPC by introduction of polymer additives was at the expense of prolonging the setting time and reducing mechanical strength and injectability of CPC. ${ }^{\mathbf{1}}$

The aim of this study is to develop a novel injectable CPC, which not only has a noticeably improved anti-washout property, but also avoids prolonging setting time and markedly reducing injectability and mechanical strength. Sodium polyacrylate (PAAS) is nontoxic and biocompatible. PAAS is widely used as a safe food additive, ${ }^{15-17}$ and has potential applications in the drug delivery or other implantable materials. ${ }^{18}$ Fig. 1 exhibits the chemical structure of PAAS. Due to the repeated carboxyl groups along the molecular chain, PAAS possesses high viscosity, strong hydrophilicity, and shape retention ability when it is dissolved in aqueous solution. Therefore, these advantages of PAAS motivated us to hypothesize that PAAS 


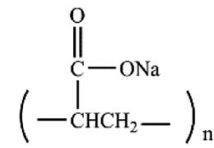

Fig. 1 The chemical structure of PAAS

could be used to improve the anti-washout property of CPC paste.

In this study, an attempt was made to improve anti-washout property of injectable CPC by introducing PAAS. The effects of PAAS on the washout resistance, injectability, rheological properties, setting time, compressive strength, porosity, phase, microstructure, and in vitro cellular behaviors were comprehensively investigated.

\section{Materials and methods}

\subsection{Material preparation}

The CPC powder used in this study was a mixture of partially crystallized calcium phosphate (PCCP), dicalcium phosphate anhydrous $\left(\mathrm{CaHPO}_{4}, \mathrm{DCPA}\right)$ and hydroxyapatite $\left(\mathrm{Ca}_{10}\left(\mathrm{PO}_{4}\right)_{6}(-\right.$ $\mathrm{OH})_{2}$, HAp) with low crystallinity. The mass ratio of PCCP, DCPA, and HAp in the mixtures was $4.5: 4.5: 1$. The preparation procedures of PCCP powder was described in the previous studies of our research group. ${ }^{19}$ PCCP powder was synthesized from an aqueous solution of $\mathrm{Ca}\left(\mathrm{NO}_{3}\right)_{2} \cdot 4 \mathrm{H}_{2} \mathrm{O}(0.36 \mathrm{M})$ and $\left(\mathrm{NH}_{4}\right)_{2} \mathrm{HPO}_{4} \cdot 12 \mathrm{H}_{2} \mathrm{O}(0.15 \mathrm{M})$ by chemical precipitation method. Then, the deposit was centrifugally separated, freeze-dried, and calcined at $450{ }^{\circ} \mathrm{C}$ for $2 \mathrm{~h}$ in a furnace, and the calcined powder was grinded to obtain the ready-for-use PCCP powder. DCPA powder was obtained by dehydration at $120^{\circ} \mathrm{C}$ for $12 \mathrm{~h}$ and then milling for $2 \mathrm{~h}$ of the commercially available dicalcium phosphate dehydrate $\left(\mathrm{CaHPO}_{4} \cdot 2 \mathrm{H}_{2} \mathrm{O}\right)$. HAp was synthesized through a chemical precipitation method using calcium nitrate $\left(\mathrm{Ca}\left(\mathrm{NO}_{3}\right)_{2} \cdot 4 \mathrm{H}_{2} \mathrm{O}, \quad 0.25 \mathrm{M}\right)$ and diammonium phosphate $\left(\left(\mathrm{NH}_{4}\right)_{2} \mathrm{HPO}_{4}, 0.15 \mathrm{M}\right) .^{20}$ Subsequently, the precipitate was washed with deionized water and centrifugally separated, lyophilized and grinded to obtain the HAp powder. PAAS powder was introduced into CPC powder at mass ratios of 0 , $0.25 \%, 0.5 \%, 0.75 \%, 1.00 \%$, and $2.00 \%$, respectively. Deionized water and CPC powder containing PAAS were homogeneously mixed in an agate mortar to obtain CPC paste with a workable consistency. The liquid to powder $(\mathrm{L} / \mathrm{P})$ ratio was $0.4 \mathrm{~mL} \mathrm{~g}^{-1}$. The cement without PAAS was used as the control group. In this study, all of the reagents used were of analytical grade and commercially obtained from a domestic company (Aladdin Industrial Corp, China) except those mentioned specially.

\subsection{Rheological properties test}

Rheological properties of CPC paste were analyzed by a rheometer (ARG2, TA Instrument, USA). The viscosity and shear stress curves were obtained by measuring the variation of viscosity and shear stress at different shear rate. During the tests, the shear rate was increased from the initial rate of $0.01 \mathrm{~s}^{-1}$ to the final rate of $100 \mathrm{~s}^{-1}$.

\subsection{Anti-washout property evaluation}

Anti-washout property evaluation of the cement pastes included qualitative visual observation and quantitative measurement. The cement paste in a syringe was immediately injected into a beaker filled with simulated body fluid (SBF, $30 \mathrm{~mL}$ ) solution. Then these samples were put into a shaker at $37^{\circ} \mathrm{C}$ and shaken at a speed of $60 \mathrm{rpm}$ for 0,15 , and $30 \mathrm{~min}$. The samples were taken out and evaluated by visual observation. For quantitative measurement, the CPC pastes were placed into stainless steel moulds. The cylindrical cement samples (diameter $=6 \mathrm{~mm}$, height $=12 \mathrm{~mm}$ ) were immediately demoulded after the moulds filled with the cement paste, and then soaked in SBF solution $(30 \mathrm{~mL}$ for each) in the beakers. The beakers with samples were fixed in the above-mentioned shaker. After being shaken for $0,10,20,30$, and $60 \mathrm{~min}$, the samples were taken out and dried at $37{ }^{\circ} \mathrm{C}$. The mass of as-prepared samples and decayed samples after shaking was measured. The anti-washout property of the cement paste was evaluated according to eqn (1). Each specimen was repeated six times.

Mass 1 oss $\%=$ (mass loss during shaking $) /($ total mass $) \times 100 \%$

\subsection{Injectability test}

Injectability of the cement paste was tested using a syringe, which is fitted with a needle of $2.1 \mathrm{~mm}$ in inner diameter. $5 \mathrm{~g}$ CPC powder was mixed with deionized water $(2.0 \mathrm{~mL})$ for $30 \mathrm{~s}$ and put into the syringe. The cement paste was extruded from the syringe by a universal material testing machine (Instron 5567 . Instron, USA) at a speed of $15 \mathrm{~mm} \min ^{-1}$ until a maximum force of $100 \mathrm{~N}$ was reached. The extrusion rate was kept constant, while the force during the extrusion process was recorded. The mass of the CPC paste inside the syringe before and after extrusion was weighed. The injectability was calculated by the mass ratio of the extruded paste to the original paste in the syringe. ${ }^{21}$ Each measurement was repeated four times.

\subsection{Setting time test}

Setting time of the cements was measured according to the Gilmore needle method. After mixing the CPC powder and liquid, the homogeneous cement paste was poured into stainless steel moulds immediately. Subsequently, the cement samples were placed in an incubator at $37{ }^{\circ} \mathrm{C}$ and $98 \%$ relative humidity. The initial setting occurred when the cement could withstand a small fixed pressure exerted by a thin Gilmore needle. The final setting took place when the thick Gilmore needle did not produce a visible impression. Each specimen was repeated four times.

\subsection{Compressive strength and porosity measurement}

A cylindric steel mould (inner diameter $=6 \mathrm{~mm}$, height $=12$ $\mathrm{mm}$ ) was used to prepare cement samples for compressive strength and porosity tests. After being poured into the moulds, the cement was pressed using a compression testing machine 
under a force about $10 \mathrm{~kg}$ for $15 \mathrm{~s}$ to remove the bubbles in the paste. The cement samples were removed from the moulds and hydrated in an incubator at $37{ }^{\circ} \mathrm{C}$ and $98 \%$ relative humidity for 3 days. A universal material testing machine (Instron 5567, Instron, USA) was used to measure the compressive strength of the cement samples at a crosshead speed of $1 \mathrm{~mm} \mathrm{~min}{ }^{-1}$. The porosity of the samples was determined by the Archimedes technique with ethanol as displacement liquid, as described in the previous report. ${ }^{6}$ Each specimen was repeated six times.

\subsection{Phase and microstructure characterization}

The hydrated samples were ground into powder, and then characterized by an X-ray diffractometer (XRD, X'Pert PRO, PANalytical, Netherlands) using $\mathrm{Cu}-\mathrm{K} \alpha$ radiation. The XRD patterns were collected in the range of $10^{\circ}$ to $70^{\circ}$ for $2 \theta$ with a step size of $0.013^{\circ}$. Microstructure and cross sectional morphology of the hydrated samples were observed under a scanning electron microscope (SEM, Nova NanoSEM 430, FEI, Netherlands) with an accelerating voltage of $15 \mathrm{kV}$ after the samples were gold-coated.

\subsection{Cytocompatibility assessment}

2.8.1. Cell culture. Mouse bone marrow stromal cells (mBMSCs, ATCC, USA) were used to examine the effect of PAAS additive on the cell behaviors on the modified CPC. mBMSCs were cultured in cell culture flasks in an incubator $\left(37^{\circ} \mathrm{C}, 5 \%\right.$ $\mathrm{CO}_{2}$ ). The complete culture medium was composed of $90 \%$ high-glucose Dulbecco's modified eagle's medium (H-DMEM; Gibco, USA) and $10 \%$ fetal bovine serum (FBS; Gibco, Hyclone, USA). The complete culture medium was refreshed every other day.

2.8.2. Cell proliferation. Six groups of hydrated cement disks (diameter $=6 \mathrm{~mm}$; height $=1.5 \mathrm{~mm}$ ) were sterilized by gamma irradiation at $15 \mathrm{kGy}$. The cement disks were placed into 48 -well plates and cell suspension $\left(500 \mu \mathrm{L}, 2 \times 10^{4}\right.$ cells per $\left.\mathrm{mL}\right)$ was added to each well. Cells seeded on the cement disks were cultured in a humidified incubator at $37{ }^{\circ} \mathrm{C}$ with $5 \% \mathrm{CO}_{2}$. The complete culture medium was refreshed every other day. Cell Counting Kit-8 (CCK-8, Dojindo Laboratories, Japan) was used to evaluate the proliferation of mBMSCs on the samples. After cell culture for 1,3 , and 7 days, the cement disks were transferred to a new 48-well plate. Then, complete culture medium $(250 \mu \mathrm{L})$ containing 10 vol\% CCK-8 work solution was added to each well and incubated at $37{ }^{\circ} \mathrm{C}$ in $5 \% \mathrm{CO}_{2}$ for $1 \mathrm{~h}$. The absorbance of supernatant was read at $450 \mathrm{~nm}$ using a microplate reader (Thermo 3001, Thermo, USA). The detected optical density (OD) values were converted to cell numbers by making a calibration curve of absorbance with cell numbers. Test for each specimen was repeated six times.

2.8.3. Cell viability and attachment. After being cultured for $24 \mathrm{~h}$, the viability of mBMSCs cultured on the CPC disks was observed using a Live kit (Biotium, USA) under a fluorescence inverted microscope (Nikon, Eclipse Ti-U, Japan), following the manufacture's instruction. Morphology of cells attached on the CPC disks was analyzed by SEM observation. After 1 day of incubation, the disks were washed three times with phosphate buffer solution (PBS), fixed with 2.5\% glutaraldehyde solution for $1 \mathrm{~h}$, followed by dehydration with a series of gradient ethanol $(30 \%, 50 \%, 70 \%, 80 \%, 90 \%$, 95\%, and 100\%), air-dried, sputtered with gold, and observed by SEM (Nova NanoSEM 430, FEI, Netherlands). Cell cytoskeleton arrangement was observed with a confocal laser scanning microscope (CLSM; TCS SP8, Leica Co., Germany). After cell culture for 1 day, the disks were taken out, washed with PBS, fixed with $4 \%$ paraformaldehyde, and immersed in $0.1 \%$ Triton (Sigma Aldrich, USA) solution for 5 min. Subsequently, the cell actin cytoskeleton and nuclei stainings were performed using FITC-phalloidin (cell navigator F-actin labelling kit, AAT Bioquest, USA) and DAPI (Beyotime, China) according to the manufacturer's protocols. Then the cell cytoskeleton was observed and photographed using CLSM.

2.8.4. Cell differentiation. mBMSCs were seeded on the cement disks with a cell density of $3 \times 10^{4}$ cells per well, and cultured in an incubator with $5 \% \mathrm{CO}_{2}$ at $37^{\circ} \mathrm{C}$. The osteoblastic differentiation of mBMSCs was assayed by the investigation of alkaline phosphatase (ALP) activity. mBMSCs were lysed using lysis buffer $(250 \mu \mathrm{L}, 0.1 \mathrm{vol} \%$ Triton X-100 in $10 \mathrm{mM}$ Tris-HCl buffer solution, $\mathrm{pH}=7.4$ ) at $4{ }^{\circ} \mathrm{C}$ for $4 \mathrm{~h}$. ALP activity was analyzed by $p$-nitrophenylphosphate ( $p$ NPP, Sigma-Aldrich, USA) method. The lysate $(20 \mu \mathrm{L})$ from each sample was added with $p$ NPP solution $(200 \mu \mathrm{L}, 5 \mathrm{mM}$, dissolved in $0.1 \mathrm{M}$ glycine buffer), incubated at $37{ }^{\circ} \mathrm{C}$ for $15 \mathrm{~min}$ and terminated by the addition of $\mathrm{NaOH}$ solution $(200 \mu \mathrm{L}, 1 \mathrm{M})$. The enzyme activity was measured based on the formation of $p$-nitrophenol $(p \mathrm{NP})$ and quantified by absorbance at $410 \mathrm{~nm}$. The total protein content of cells was assessed using BCA Protein Assay Kit (Thermo Scientific, USA) according to the manufacturer's protocol and absorbance at $562 \mathrm{~nm}$ was read. The ALP activity was expressed as enzyme activity unit per milligram of total protein content.

\subsection{Statistical analysis}

All data in this study were presented as mean value \pm standard deviation (SD). One-way analysis of variance (one-way ANOVA) was used to evaluate the significant differences between the experimental data. A value of $p<0.05$ was considered as statistical significance $(* p<0.05, * * p<0.01, * * * p 0.001)$.

\section{Results and discussion}

\subsection{The preparation of anti-washout CPC}

In this study, we have fabricated a novel anti-washout CPC for bone repair by the addition of biocompatible PAAS, and investigated the effect of PAAS content on the physical-chemical properties and in vitro cellular behaviors of CPC. PAAS is a water soluble polymer, so it will dissolve when mixing the CPC powder containing PAAS with water and increase the viscosity and cohesion of the CPC pastes. On one hand, PAAS could serve as an anti-washout agent to enhance the anti-washout property of CPC. On the other hand, PAAS could increase the viscosity of the cement paste and the stability of the CPC pastes with an appropriate content of PAAS, thus endowing the cement with an excellent injectability. 


\subsection{Rheological properties}

The rheological properties of CPC pastes with different PAAS contents are shown in Fig. 2. As shown in Fig. 2(a), when the shear rate was lower than $0.8 \mathrm{~s}^{-1}$, the viscosity of all the CPCs with and without PAAS decreased rapidly with the increase of shear rate. When the shear rate was higher than $0.8 \mathrm{~s}^{-1}$, the viscosity of CPC pastes declined at first, and then tended to be in a steady state. The viscosity of CPCs increased with the increase of PAAS content. Fig. 2(b) reveals that the shear stress of CPC pastes increased with the increase of PAAS content. At the shear rate of $40 \mathrm{~s}^{-1}$, it increased from $22 \mathrm{~Pa}$ to $537 \mathrm{~Pa}$ as the PAAS content increased from 0 to $2.00 \mathrm{wt} \%$. When the shear rate was higher than $40 \mathrm{~s}^{-1}$, the shear stress of CPC pastes tended to be stable. The viscosity and shear stress of the cement containing $2.00 \mathrm{wt} \%$ PAAS was much higher than those of the cements with lower PAAS contents.

\subsection{Anti-washout property}

The qualitative and quantitative results of anti-washout property of the cements with different concentrations of PAAS are shown in Fig. 3 and 4, respectively. The mass loss of all the groups increased with the prolongation of shaking time. The control cement paste in the SBF solution could not maintain an intact shape of long strip and its mass loss reached $63.26 \% \pm$ $2.95 \%$ after shaking for $1 \mathrm{~h}$. There was no noticeable disintegration happening to all the CPC samples modified with PAAS before shaking. As the content of PAAS in CPC increased from $0.25 \mathrm{wt} \%$ to $2.00 \mathrm{wt} \%$, the mass loss of CPC paste decreased from $10.23 \% \pm 1.48 \%$ to $4.97 \% \pm 0.84 \%$ after shaking for $1 \mathrm{~h}$.

In this study, PAAS effectively improved anti-washout property of CPC. As the hydration reaction happens, the surrounding water is likely to invade the cement paste, destroying the connection among the CPC particles or HAp crystals and leading to decay of cement paste. ${ }^{22,23}$ It is known that PAAS has excellent hydrophilicity and gel properties. ${ }^{24}$ When PAAS was introduced into CPC system, a network spreading all over the paste could form and immobilize the CPC particles, maintaining the shape of paste even exposed to the surrounding liquid. Some studies demonstrated that the anti-washout property of CPC is often associated with cohesion. ${ }^{1}$ Baroud et al. ${ }^{25}$ proposed that using high-viscosity cement could effectively prevent extraosseous cement leakage, which often occurs during the vertebroplasty procedures. The viscosity of CPC paste increased with the increase of PAAS content (Fig. 2(a)). PAAS acted as
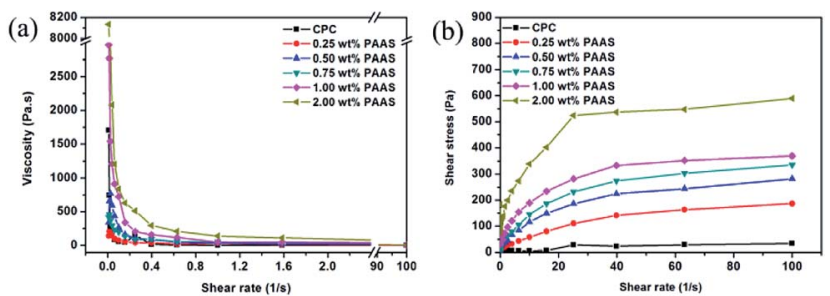

Fig. 2 Influence of PAAS content on the viscosity (a) and shear stress (b).

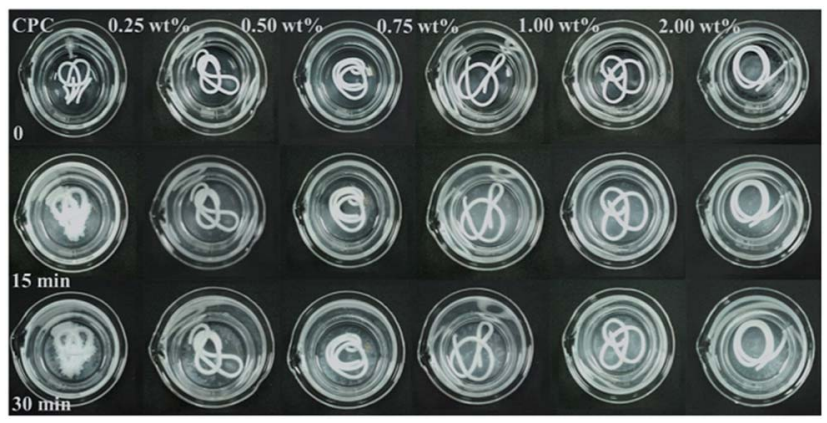

Fig. 3 Anti-washout observation of cement pastes in SBF after shaking for 0,15 , and $30 \mathrm{~min}$.

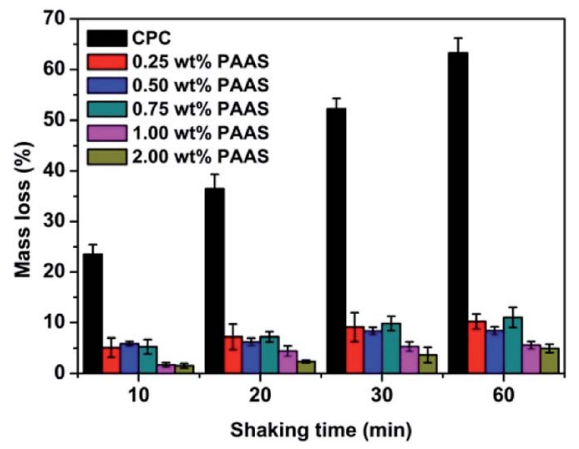

Fig. 4 Mass loss of cement pastes in SBF after shaking for 0, 10, 20, 30 , and $60 \mathrm{~min}$.

a binder to increase the cohesion of the cement paste, restraining the penetration of liquid into the cement paste and the separation of the CPC particles and decreasing the mass loss during shaking. In addition, it was reported that PAAS formed insoluble gelatinous calcium salt by cross-linking of carboxyl groups with $\mathrm{Ca}^{2+} \cdot{ }^{26}$ The PAAS molecules adsorbed on the surface of CPC particles via chemical bonding between carboxyl groups in PAAS and calcium ions in CPC, which promoted the CPC particles interlocking. As a result, the antiwashout ability of CPC was markedly enhanced by the addition of PAAS.

\subsection{Injectability}

With the increasing development of minimally invasive techniques in surgical procedures, there is growing requirement in the injectability of CPC. Fig. 5(a) shows the injectability of the CPCs with and without PAAS. Except the control CPC paste $(36.37 \% \pm 4.30 \%)$, all the cements with PAAS were almost completely extruded. Compared with other anti-washout agents, such as chitosan and sodium alginate, the addition of PAAS is more beneficial to improve the injectability of CPC while enhancing its anti-washout property.

The control group showed poor injectability, which was reflected by severe solid-liquid separation during the extrusion, as described in previous report. ${ }^{27}$ It can be seen that the significant improvement in the injectability of cement paste by the addition of PAAS was attributed to its excellent rheological 

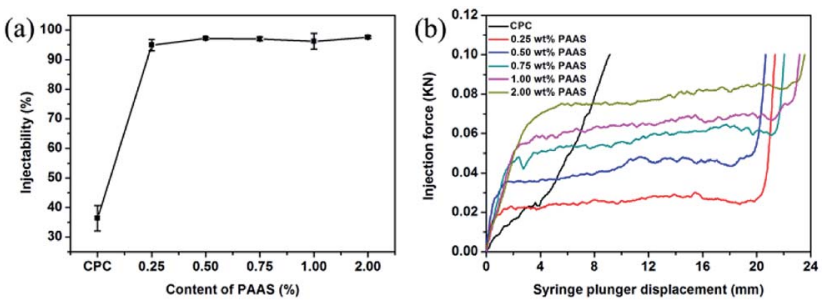

Fig. 5 The injectability (a) and extrusion curves (b) of the cements with different contents of PAAS.

properties and stability. The cement pastes with PAAS were strongly shear-thinning, and could simultaneously behave like a very viscous paste. Moreover, PAAS was used to modify the injectability via a chemical interaction. PAAS is indeed known to interact with calcium ions ${ }^{27,28}$ and is often used to increase the electronegativity of ceramic slips and improve the rheological properties as a dispersant. The dispersing performance of PAAS depends on its degree of polymerization.

Fig. 5(b) shows the extrusion curves recorded during the injection processes. For the control CPC, there was a sharp monotonous increase in the extrusion force, which could be attributed to phase separation. By contrast, the extrusion force of CPC pastes with PAAS was typically characterized by an initial accelerated increase stage, a subsequent smooth plateau stage, and a final rapid increase stage. The accelerated extrusion force at the initial stage was caused by the yield stress that must be applied to make the paste start flowing. The smooth plateau of the extrusion force was caused by requirement of maintaining the paste flow. From these curves, it was apparent that the extrusion force at the plateau increased from $20 \mathrm{~N}$ to $80 \mathrm{~N}$ when the content of PAAS increased from $0.25 \mathrm{wt} \%$ to $2.00 \mathrm{wt} \%$. This was attributed to the elevated viscosity of the pastes when a higher content of PAAS was introduced. At the end of the extrusion, the rapid increase in the extrusion force resulted from the mechanical contact between the syringe plunger and the bottom of syringe when almost all the paste was extruded.

\subsection{Setting time}

A suitable setting time is important for injectable cement in clinical application. Reports indicated that some polymers could influence the hydration process of CPC and prolong its setting time. ${ }^{29-31}$ Longer setting time is not conducive to improvement of anti-washout property. The effect of PAAS content on the setting time of CPC is shown in Fig. 6. It can be seen that the addition of PAAS did not significantly affect the initial and final setting time of cements when the content of PAAS was no more than $1 \mathrm{wt} \%$. However, the addition of $2 \mathrm{wt} \%$ PAAS slightly prolonged the final setting time of CPC. The PAAS adsorbed on the surface of cement particles, which slowed down hydration process and then prolonged the setting time of CPC.

\subsection{Compressive strength and porosity}

Fig. 7 presents the compressive strength of the cement samples. Low content of PAAS ( $\leq 1.00 \mathrm{wt} \%)$ decreased slightly the

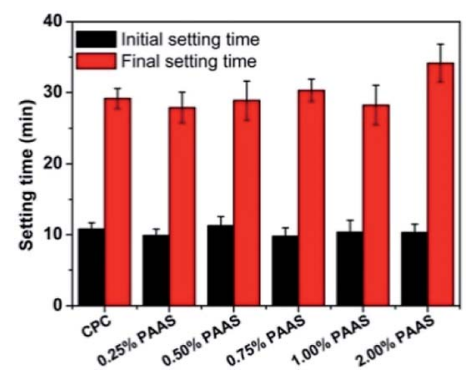

Fig. 6 Influence of PAAS content on the initial and final setting time of the cements.

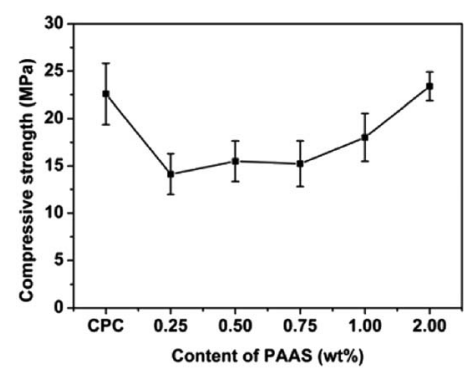

Fig. 7 Influence of PAAS content on the compressive strength of the cements.

compressive strength of CPC compared with the control group $(22.59 \pm 2.52 \mathrm{MPa})$. When the PAAS content increased from 0.25 to $2.00 \mathrm{wt} \%$, the compressive strength of CPC increased from $14.13 \pm 0.62 \mathrm{MPa}$ to $23.39 \pm 2.57 \mathrm{MPa}$.

Generally speaking, smaller amount of PAAS adsorbed on the surface of CPC particles would weaken interface between CPC particles and result in the decrease of compressive strength. Chen et $a l .{ }^{32}$ found the similar results that a higher weight ratio of polyacrylic acid to CPC was feasible to increase the compressive strength of CPC samples. In this study, larger amount of PAAS in CPC matrix would tend to form a homogeneous gel with three dimensional network structure, wrapping cement particles together by forming cross-links between carboxyl groups and calcium ions. This would contribute to the increase in compressive strength of CPC when the PAAS content increased.

The porosity of cement samples is shown in Fig. 8. The addition of PAAS had little effect on the porosity of CPC, and the porosity stayed between $40 \%$ and $50 \%$.

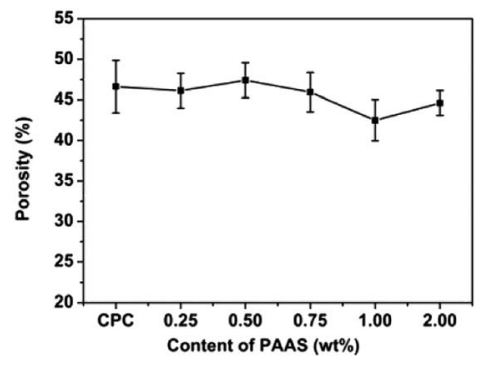

Fig. 8 Influence of PAAS content on the porosity of the cements. 


\subsection{Phase and microstructure characterization}

Fig. 9 shows XRD patterns of the CPCs with different concentrations of PAAS after hydration for 3 days. The crystallization peaks were attributed to poorly crystalline HAp (JCPDS card no. 09-0432) resulting from the dissolution-deposition reaction between PCCP and DCPA. ${ }^{19}$ There was no obvious difference in the XRD patterns among the various experimental groups. Fig. 10 presents SEM photographs of the fractured surface of the control CPC and the samples with different concentrations of PAAS. It can be observed that a lot of fine needle-like and platelike apatite grains interlocked with each other. Plentiful nano or submicron pores with irregular shapes were observed. This illustrated that the addition of PAAS did not significantly influence the hydration product and microstructure of CPC.

\subsection{In vitro biocompatibility}

PAAS is known to be nontoxic as a sodium salt of PAA, which has been used in the drug delivery field. Fig. 11(a) presents the morphology of cell attached on the CPC samples after cell culture for 1 day. The cells completely spread on all the samples with outstretched pseudopodium, indicating good cell adhesion to the surfaces of all the CPCs. The images of nuclear (blue) and cytoskeleton (green) of the cells cultured on disks are shown in Fig. 11(b). The cells were in spindle-shape and well spread. A large number of filopodia-like pseudopods were exhibited on the surface of cells. Moreover, cells attaching on the surfaces of CPC samples were closely connected with each other. Fig. 11(c) displays the fluorescence photographs of

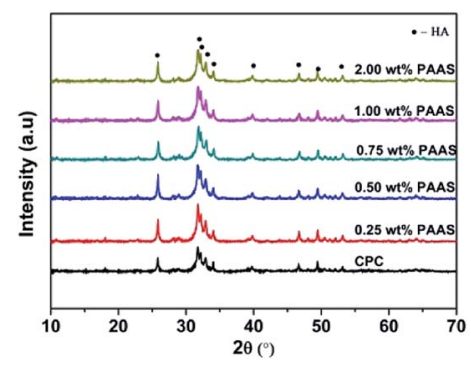

Fig. 9 XRD patterns of the hydrated cements.
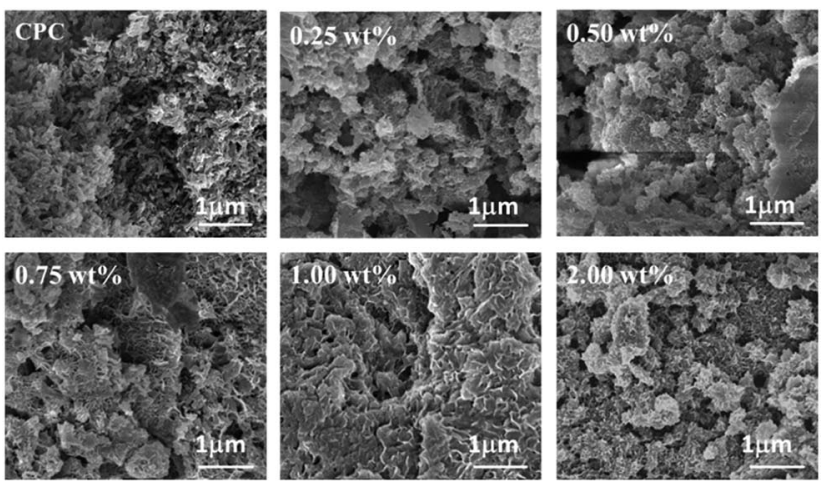

Fig. 10 SEM micrographs of the hydrated cements.

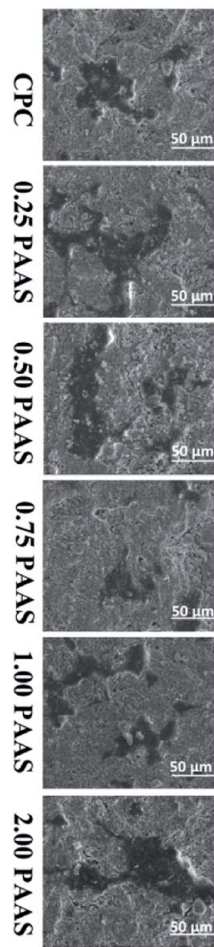

(a)
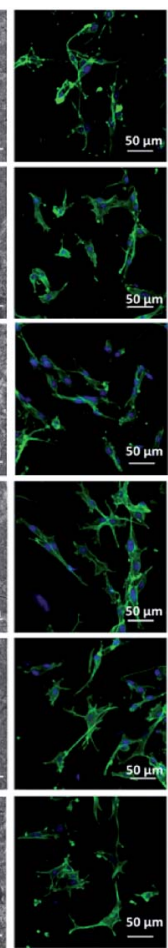

(b)
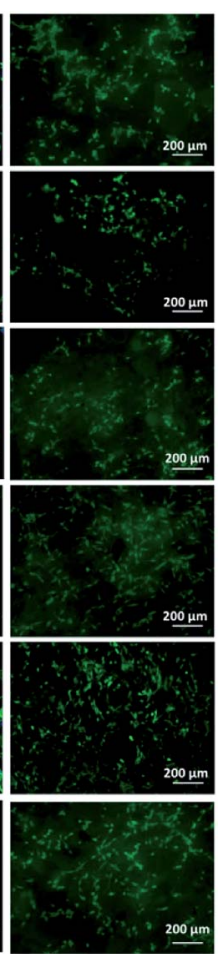

(c)
Fig. 11 Attachment morphology (a), nuclear and cytoskeleton staining (b), fluorescence photographs (c) of mBMSCs on CPCs after cultured for 1 day. They were evaluated by SEM, confocal laser scanning microscope, and fluorescence inverted microscope, respectively.

mBMSCs cultured on the CPC samples. The cells grew and spread very well. It suggested that the introduction of PAAS was beneficial for cell adhesion.

The cell proliferation on the CPC samples is presented in Fig. 12. CCK-8 assay indicated that the proliferation of mBMSCs on the CPCs with PAAS $(\leq 1.00 \mathrm{wt} \%)$ was superior to that on the control CPC after cell culture for 3 and 7 days. When the content of PAAS was up to $2 \mathrm{wt} \%$, the proliferation of cells was significantly inhibited on the day 3 but had no obvious variation on the day 7 compared with the control group.

ALP activity is deemed as an early marker of osteogenic differentiation. The ALP activity of mBMSCs on the CPC samples is shown in Fig. 13. The ALP activity of mBMSCs on the samples of all the groups had no obvious variation on the day 7 .

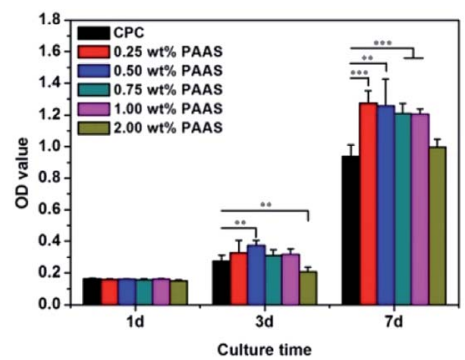

Fig. 12 The proliferation of mBMSCs cultured on CPC and CPC with PAAS. Data are represented as means \pm SD $(* * p<0.01, * * * p<0.001)$. 


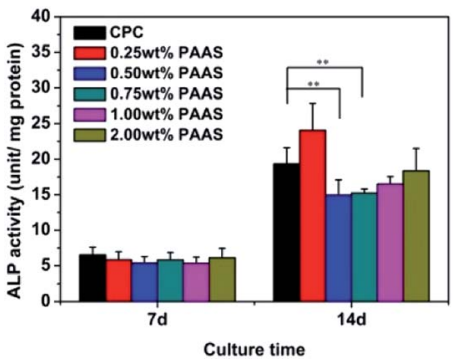

Fig. 13 ALP activity of mBMSCs cultured on the cement after 7 and 14 days. Data are represented means \pm SD $(* p<0.05, * * p<0.1)$.

On the day 14, the ALP activity of mBMSCs on the sample with 0.25 wt $\%$ PAAS was slightly higher than that on the control group, but no statistical significance was attained. When the content of PAAS was more than $0.25 \mathrm{wt} \%$, the ALP activity was a little lower than that on the control group.

Based on the mBMSCs responses to the cement samples, all the CPCs with and without PAAS were demonstrated to provide favourable conditions for cell adhesion, spreading, and proliferation, proving the biocompatibility of PAAS as an effective antiwashout agent. The cell behaviors of CPC samples might have something to do with different surface conditions, such as hydrophilicity and surface electrostatic. Due to the repeated carboxyl groups along the molecular chain and strong hydrophilicity, PAAS might enhance the surface hydrophilicity, electronegativity, and protein adsorption of CPC. This contributed to the cell proliferation. Previous studies ${ }^{33,34}$ reported that poly(acrylic acid) surface with negative charges promoted the cell adhesion, proliferation, osteogenic differentiation, and gene expression of the MSCs by introducing carboxyl. In these studies, PAA-modified surfaces adsorbed more fibronectin molecules, whose conformation might affect its specific bonding with integrin. Moreover, the surface density of the PAA molecules affected protein adsorption, cell adhesion, and proliferation..$^{\mathbf{3 4 3 5}}$

\section{Conclusions}

In this study, an anti-washout injectable CPC for bone repair was successfully fabricated by the addition of PAAS. It is indicated that the addition of PAAS significantly improve the washout resistance and injectability of CPC. PAAS hardly affected the transformation to Hap during hydration process, setting time, microstructure, and morphology of CPC. Low content of PAAS slightly reduced the compressive strength, but the compressive strength of CPC gradually increased with the increasing PAAS content. Moreover, the modified CPC containing PAAS was proved to be biocompatible and support adhesion and proliferation of mBMSCs. Therefore, the antiwashout cement containing PAAS has the potential for use in orthopedic applications.

\section{Acknowledgements}

The authors gratefully acknowledge the financial supports to this research work from the National High Technology Research and Development Program of China under Grant No. 2015AA033601, the National Natural Science Foundation of China under Grant No. 51672087, and the Science and Technology Program of Guangzhou City of China under Grant No. 201508020017.

\section{Notes and references}

1 J. T. Zhang, W. Z. Liu, V. Schnitzler, F. Tancret and J. M. Bouler, Acta Biomater., 2014, 10, 1035-1049.

2 S. M. Barinov and V. S. Komlev, Inorg. Mater., 2011, 47, 14701485.

3 F. P. Chen, Z. Y. Song and C. S. Liu, J. Mater. Chem. B, 2015, 3, 9173-9181.

4 U. Gbureck, J. E. Barralet, K. Spatz, L. M. Grover and R. Thull, Biomaterials, 2004, 25, 2187-2195.

5 E. F. Ahmed, J. H. Kim, R. A. Perez and H. W. Kim, J. Mater. Chem. B, 2015, 3, 1321-1334.

6 J. Q. Liu, J. Y. Li, J. D. Ye and F. P. He, Ceram. Int., 2016, 42, 13670-13681.

7 K. Ishikawa, Y. Miyamoto, M. Takechi, T. Toh and M. Kon, J. Biomed. Mater. Res., 1996, 36, 393-399.

8 M. Takechi, Y. Miyamoto, K. Ishikawa, M. Yuasa, M. Nagayama, M. Kon and K. Asaoka, J. Mater. Sci.: Mater. Med., 1996, 7, 317-322.

9 A. Yokoyama, S. Yamamoto, T. Kawasaki, T. Kohgo and M. Nakasu, Biomaterials, 2002, 23, 1091-1101.

10 X. P. Wang, L. Chen, H. Xiang and J. D. Ye, J. Biomed. Mater. Res., Part B, 2008, 81, 410-418.

11 A. Cherng, S. Takagi and L. C. Chow, J. Biomed. Mater. Res., 1997, 35, 273-277.

12 W. Z. Liu, J. T. Zhang, G. Rethore, K. Khairoun, P. Pielet, F. Tancret, J. M. Bouler and P. Weiss, Acta Biomater., 2014, 10, 3335-3345.

13 J. T. Zhang, W. Z. Liu, O. Gauthier, S. Sourice and P. Pilet, Acta Biomater., 2016, 31, 326-338.

14 F. Wu, Y. Ngothai, J. Wei, C. S. Liu, B. O'Neil and Y. Q. Wu, Colloids Surf., B, 2011, 92, 113-120.

15 Y. Liu, Y. Sun, L. F. Sun, R. Rehman and Y. P. Wang, J. Funct. Foods, 2016, 24, 429-437.

16 S. Anlar, Y. Capan and A. Hincal, Die Pharmazie, 1993, 48, 285-287.

17 M. A. Longer, H. S. Chng and J. P. Robinson, J. Pharmaceut. Sci., 1985, 74, 406-411.

18 X. Y. Ma, L. J. Shi, J. Zhou, J. Zhu, J. Zhong and R. L. Wei, Chin. J. Org. Chem., 2013, 33, 1080-1087.

19 X. P. Wang, J. D. Ye and Y. J. Wang, J. Mater. Sci.: Mater. Med., 2008, 19, 813-816.

20 I. Mobasherpour, M. S. Heshajin, A. Kazemzadeh and M. Zakeri, J. Alloys Compd., 2007, 430, 330-333.

21 R. M. O'hara, N. J. Dunne, J. F. Orr, F. J. Buchanan, R. K. Wilcox and D. C. Barton, J. Mater. Sci.: Mater. Med., 2010, 21, 2299-2305.

22 D. Kai, D. X. Li, X. D. Zhu, L. Zhang, H. S. Fan and X. D. Zhang, J. Mater. Sci.: Mater. Med., 2009, 20, 1595-1602.

23 K. Ishikawa, Y. Miyamoto, M. Kon, M. Nagayama and K. Asaoka, Biomaterials, 1995, 16, 527-532. 
24 Y. Lu and T. Li, Biomaterials, 2009, 30, 4143-4151.

25 G. Baroud, M. Crookshank and M. Bohner, Spine, 2006, 31, 2562-2568.

26 N. Yoshiyuki, N. Satoshi, O. Seiichi and I. Taichi, Carbohydr. Polym., 2015, 117, 43-53.

27 M. Bohner and G. Barroud, Biomaterials, 2005, 26, 15531563.

28 W. N. Charman, E. P. Geunin and D. C. Monkhouse, Drug Dev. Ind. Pharm., 1991, 17, 271-280.

29 M. H. Alkhraisat, C. Rueda, F. T. Mariño, J. Torres, L. B. Jerez, U. Gbureck and E. L. Cabarcos, Acta Biomater., 2009, 5, 31503156.
30 J. An, G. C. Joop and A. John, J. Mater. Sci.: Mater. Med., 2016, 27, 58-66.

31 J. Q. Liu, J. Y. Li and J. D. Ye, J. Mater. Sci. Technol., 2016, 32, 1021-1026.

32 W. C. Chen, C. P. Ju, J. C. Wang, C. C. Hung and J. H. Chern Lin, Dent. Mater., 2008, 24, 1616-1622.

33 J. M. Curran, R. Chen and J. A. Hunt, Biomaterials, 2005, 26, 7057-7067.

34 L. K. Guo, T. Hoshiba, T. Tateishi, G. P. Chen and X. D. Zhang, J. Biomed. Mater. Res., Part A, 2008, 87, 903-912.

35 B. Li, Y. Ma, S. Wang and P. M. Moran, Biomaterials, 2005, 26, 4956-4963. 